\title{
A novel DAX-1 (NR0B1) mutation in a boy with X-linked adrenal hypoplasia congenita
}

\author{
Gerster, Karine ; Katschnig, Claudia ; Wyss, Sascha ; Kolly, A ; Sproll, P ; Biason-Lauber, Anna ;
} Konrad, Daniel

\begin{abstract}
BACKGROUND: X-linked adrenal hypoplasia congenita (AHC) is caused by mutations in DAX-1 (NR0B1) playing a key role in adrenal and reproductive development. CASE PRESENTATION: Herein we report a 2.5-year-old boy who presented with acute adrenal failure. Family history revealed unexplained death in three brothers of the patient's mother during infancy. Molecular analysis of the DAX-1 gene revealed the presence of a novel hemizygous mutation, c. $870 \mathrm{C}>\mathrm{A}$ in exon 1 , leading to the formation of a premature stop codon. The same mutation was identified in the patient's mother. The truncated mutant protein is most likely misfolded, sequestered in the endoplasmic reticulum and therefore cannot bind to and activate its target DNA sequences in the nucleus. CONCLUSIONS: DAX-1 mutation must be considered when diagnosis of primary adrenocortical insufficiency is made, especially if there is a history of unexplained death of maternal male relatives.
\end{abstract}

DOI: https://doi.org/10.1515/jpem-2017-0261

Posted at the Zurich Open Repository and Archive, University of Zurich

ZORA URL: https://doi.org/10.5167/uzh-143639

Journal Article

Accepted Version

Originally published at:

Gerster, Karine; Katschnig, Claudia; Wyss, Sascha; Kolly, A; Sproll, P; Biason-Lauber, Anna; Konrad, Daniel (2017). A novel DAX-1 (NR0B1) mutation in a boy with X-linked adrenal hypoplasia congenita. Journal of Pediatric Endocrinology Metabolism, 12(30):1321-1325.

DOI: https://doi.org/10.1515/jpem-2017-0261 


\section{A novel DAX-1 (NR0B1) mutation in a boy with X-linked adrenal hypoplasia congenita}

Karine Gerster ${ }^{1}$, Claudia Katschnig ${ }^{1}$, Sascha Wyss ${ }^{3}$, Anne Kolly ${ }^{3}$, Patrick Sproll ${ }^{3}$, Anna

Biason-Lauber ${ }^{3,4}$, Daniel Konrad ${ }^{1,2,4}$

${ }^{1}$ Department of Endocrinology and Diabetology and ${ }^{2}$ Children's Research Center, University Children's Hospital, Zurich

${ }^{3}$ Department of Medicine, University of Fribourg, Switzerland

${ }^{4}$ Shared last authorship

Abbreviated Title: Novel DAX-1 mutation

Key words: DAX-1, X-linked adrenal hypoplasia congenital, adrenal insufficiency

\section{Corresponding Authors:}

Daniel Konrad, MD PhD

Department of Endocrinology and Diabetology

University Children's Hospital

Steinwiesstrasse 75

8032 Zurich, Switzerland

Phone: +4144266 7309

Fax: +4144266 7983

e-mail: daniel.konrad@kispi.uzh.ch

and/or: 
Anna Biason-Lauber, MD

Department of Medicine, Division of Endocrinology

University of Fribourg

Chemin du Musée 5

1700 Fribourg

Switzerland

Phone: + 41263008534

e-mail: anna.lauber@unifr.ch 


\section{Abstract (139/150)}

Background: X-linked adrenal hypoplasia congenita $(\mathrm{AHC})$ is caused by mutations in DAX-1 (NR0B1) playing a key role in adrenal and reproductive development.

Case Presentation: Herein we report a 2.5-year-old boy who presented with acute adrenal failure. Family history revealed unexplained death in 3 brothers of the patient's mother during infancy. Molecular analysis of the DAX1 gene revealed the presence of a novel hemizygous mutation, $\mathrm{c} .870 \mathrm{C}>\mathrm{A}$ in Exon 1, leading to the formation a premature stop codon. The same mutation was identified in the patient's mother. The truncated mutant protein is most likely misfolded, sequestered in the endoplasmic reticulum and therefore cannot bind to and activate its target DNA sequences in the nucleus.

Conclusions: DAX-1 mutation must be considered when diagnosis of primary adrenocortical insufficiency is made, especially if there is a history of unexplained death of maternal male relatives. 


\section{Introduction}

Hypoplasia/agenesis of adrenals leading to acute adrenal insufficiency with saltlosing crisis as a fatal condition was first reported by Sikl in 1948 (1). In the 1990's specific mutations of DAX-1 (Dosage-sensitive sex reversal - Adrenal hypoplasia congenital critical region on the $\mathrm{X}$ chromosome 1 , known also as NR0B1 (OMIM 300473)), situated in Xp21, were described for the first time to cause X-linked adrenal hypoplasia congenita (AHC) (OMIM 300200). At that time, DAX-1 was recognized to play a key role not only in the development of the adrenal gland, but also in the development of the testis, ovary, pituitary gland and hypothalamus. These first reports linked X-linked AHC to glycerol kinase deficiency (GKD) and Duchenne muscular dystrophy (DMD), which helped to discover the localisation of the gene and its mutations causing X-linked AHC. Since then a wide phenotypic spectrum has been described, patients being mostly affected by frameshift or nonsense mutations that cause premature truncation of the protein.

DAX-1encodes an orphan nuclear hormone receptor and interacts as a dominant negative regulator with other nuclear receptors, such as SF-1 to regulate gene expression (2). However, its exact biological role remains mainly unclear. In mice, deletion of exon 2 in DAX-1 leads to impaired spermatogenesis but normal adrenal function (3). Interestingly, duplications of DAX-1 have been described in $46, \mathrm{XY}$ disorder of sexual development (DSD). Possibly, this could mean that over-expression of DAX-1 suppresses male sexual development (4).

Clinically, most males with X-linked AHC present with acute adrenal failure. Adrenal insufficiency with salt-losing crisis, which is lethal if untreated, can present either in the

first 2 months of life or more gradually during childhood (5). Pubertal development is usually absent. However, some affected boys might develop pubertal sign up to Tanner 
stage 3 , when puberty stops due to disrupted hypothalamic and pituitary release of gonadotropin-releasing hormone and gonadotropins, respectively (6). Similarly, a few patients with DAX-1 mutation were reported to develop ACTH-dependent transient precocious pseudopuberty during infancy/childhood $(7,8)$.

Herein, we present a novel DAX-1 mutation leading to X-linked AHC with acute adrenal failure in a 2.5-year-old boy.

\section{Case Presentation}

A 2.5-year-old boy who presented with vomiting and severe slackness was admitted to the emergency department. Physical examination revealed moderate dehydration, underweight (weight: $11 \mathrm{~kg}$ (Z-score -1.92), length: $92 \mathrm{~cm}$ (Z-score -0.57), normal developed male genitalia and hyperpigmented lips and nipples. Laboratory evaluation revealed hypoglycaemia $(2.5 \mathrm{mmol} / \mathrm{l})$, hyponatraemia $(121 \mathrm{mmol} / \mathrm{l})$, hypochloraemia $(97$ $\mathrm{mmol} / \mathrm{l})$, hyperkalaemia (4.8 mmol/l), metabolic acidosis ( $\mathrm{pH} 7.33, \mathrm{BE}-11.9)$, low cortisol (1 nmol/l) and elevated ACTH $(6040 \mathrm{pg} / \mathrm{ml})$ concentration. Insulin was suppressed and growth hormone appropriately elevated ruling out other endocrine aetiologies for hypoglycaemia. A diagnosis of adrenal insufficiency was made and the boy was transferred to the intensive care unit for rehydration and substitution with hydrocortisone and sodium. After stabilization of vital parameters, the patient was switched to oral supplementation with hydrocortisone $\left(14 \mathrm{mg} / \mathrm{m}^{2} /\right.$ day $)$ and fludrocortisone $(0.1 \mathrm{mg} /$ day $)$. The boy was discharged from hospital after a couple of days. Growth and psychomotor development have been normal since then. At 4 years of age, his weight is $17 \mathrm{~kg}$ (Z-score $0.44)$ and height is $102.5 \mathrm{~cm}$ (Z-score 0.03$)$. 
The boy was born after normal pregnancy. Neonatal period was uneventful. He had a history of recurrent vomiting without diarrhea and increasing hyperpigmentation of the skin over the last 6 months before admission. Psychomotor development was reported to be normal. Family history revealed unexplained deaths in 3 brothers of the mother during infancy.

On additional laboratory evaluation, congenital adrenal hyperplasia (low 17hydroxyprogesterone) and adrenoleukodystrophy (normal plasma concentration of very long-chain fatty acids) could be excluded. Negative anti-21-hydroxylase and anti-adrenal autoantibodies made an autoimmune Addison disease very unlikely. Given the family history DAX-1 mutation was assumed and a molecular analysis initiated.

To this end, genomic DNA was extracted from peripheral blood leukocytes of the patient and the 2 exons of DAX1 (RefSeq NG_009814.1) were amplified and sequenced (conditions available upon request). Direct sequencing of the PCR fragments revealed the presence of a novel hemizygous mutation, c.870C $>\mathrm{A}$ in Exon 1, leading to the substitution of a cystein at position 290 with a premature stop codon (p.Cys290Ter; Figure 1). The parents were also screened and his mother was shown to be carrier of the same mutation, as to be expected for a X-linked condition.

To further analyze the molecular mechanism of the complete loss of function of the nonsense mutations, we explored the possibility of Nonsense-Mediated Decay (NMD) by stabilizing mRNA through the translation inhibitor cycloheximide. We demonstrated the loss-of-function of DAX1 is not due toNMD, as shown by the lack of differences in mRNA stability between WT and mutant DAX1under cycloheximide treatment (data not shown). Furthermore, Western Blot analysis showed no decrease in mutant protein quantity or stability, although the detected protein was, as expected, shorter (data not shown). 
Molecular models of the wild type and mutant protein were created with the online tool I-TASSER, based on homology modelling. Furthermore, the model was refined with a 5 ns refinement molecular dynamics run using YASARA (yasara.org) and finally depicted using PyMol (pymol.org). Thep.Cys290 stop mutation lead to a C-terminal truncation of 180 amino acids from DAX1 (Figure 2). The homology modelling predicts that the folding of the mutant DAX1 is disrupted and, hence, significantly different compared to the predicted protein folding of the WT DAX1. Accordingly, immunofluorescence experiments performed in transfected COS-1 cells showed that whereas WT DAX1 correctly concentrated in the nuclei, the mutant protein was detectable only in the cytoplasm of transfected COS1 cells, and is therefore prevented to exert its function as a transcription factor thus leading to loss-of-function and disease (data not shown).

\section{Discussion}

X-linked adrenal hypoplasia congenita is caused by mutations in DAX1 gene. To date even though over one hundred individuals and relatives with X-linked AHC have been reported in the literature, the exact prevalence of NROB1 mutations causing adrenal insufficiency is not clear. Studies suggest an occurrence of X-linked AHC of around $1: 140,000$ to $1: 1,200,000$ children (or between $1: 70,000$ and $1: 600,000$ males) (5).

Lin et al reported finding DAX1 mutations in all patients with adrenal insufficiency of their cohort (8 cases of a cohort of 117 patients) when family history revealed adrenal failure or unexpected death in males together with a history of arrested or absent puberty. This underlines the importance of taking an exact family history suggesting any insight into possible adrenal disease (5). In line with such findings and the fact that DAX-1 mutations follow an X-linked pattern of inheritance, family history of three maternal male 
relatives dying during neonatal period was suggestive for DAX-1 defect being retrospectively the putative cause of their early death.

Genetic analysis revealed a novel hemizygous mutation, c.870C>A, in Exon 1. This mutation led to the formation of a premature stop codon resulting in a C-terminal truncation of 180 amino acids from DAX1. The DAX1 gene encodes a protein that belongs to the nuclear hormone receptor superfamily with an amino-terminal region and a carboxylterminal region (2). Nonsense and frameshift mutations can be located throughout the NR0B1 gene. Missense mutations tend to be more prevalent in certain regions of the ligand-like binding domain, in highly conserved amino acids (9). The $\mathrm{C}$ terminus shows characteristics of a nuclear hormone receptor ligand-binding domain. In 1997, Lalli et al. discovered that the DAX1 $\mathrm{C}$ terminus contains transcriptional silencing activity, which can be transferred to a heterologous DNA-binding domain (10). Nakae found that especially mutations in the $\mathrm{C}$-terminus of the DAX-1 protein cause AHC. They postulated that the $\mathrm{C}$ terminal domain of the DAX-1 protein is important for normal adrenal gland development. Even impairment of 11 amino acids in this region can lead to disrupted adrenal cortical differentiation (11).

Phenotypes in patients harbouring a DAX 1 mutation show wide heterogeneity. As there is no genotype-phenotype correlation, DAX-1 mutation does not predict which clinical characteristics can be expected. Moreover, age of onset of $\mathrm{AHC}$ and the position of the mutation do not correlate (11). Within the same family harbouring the same mutation onset of clinical manifestation can vary. This may also be suggested in our family where manifestation of adrenal insufficiency in our patient occurred only in the third year compared to his uncles with suggested DAX1 mutation who died in the first year. Such findings suggest various degrees of penetrance due to co-modulating and/or epigenetic 
or nongenetic factors influencing DAX-1 activity and integrity and thereby the clinical course of $\mathrm{AHC}(12)$.

In conclusion, we identified a novel hemizygous mutation in a patient with X-linked AHC. This mutation led to the formation of a premature stop codon, resulting in a Cterminal truncation of 180 amino acids from DAX1, which is probably misfolded, sequestered in the endoplasmic reticulum and most likely degraded. Differentiating adrenal insufficiency due to DAX-1 mutation from other causes is of great importance since it has implications for future follow-up and treatment as well as family planning and counselling.

\section{Acknowledgements}

The work was supported by the Swiss National Science Foundation Grant Nr. $320030-130645$ to A. B-L.

\section{Statement of Ethics}

The patient's parents gave their full consent for genetic testing.

\section{Disclosure Statement}

The authors report no conflict of interest and have nothing to disclose. 


\section{References}

1. Sikl H. Addison's disease due to congenital hypoplasia of the adrenals in an infant aged 33 days. J Pathol Bacteriol. 1948;60(2):323.

2. Iyer AK, McCabe ER. Molecular mechanisms of DAX1 action. Mol Genet Metab. 2004;83(1-2):60-73.

3. Yu RN, Ito M, Saunders TL, Camper SA, Jameson JL. Role of Ahch in gonadal development and gametogenesis. Nat Genet. 1998;20(4):353-7.

4. Bardoni B, Zanaria E, Guioli S, Floridia G, Worley KC, Tonini G, et al. A dosage sensitive locus at chromosome Xp21 is involved in male to female sex reversal. Nat Genet. 1994;7(4):497-501.

5. Lin L, Gu WX, Ozisik G, To WS, Owen CJ, Jameson JL, et al. Analysis of DAX1 (NR0B1) and steroidogenic factor-1 (NR5A1) in children and adults with primary adrenal failure: ten years' experience. J Clin Endocrinol Metab. 2006;91(8):3048-54.

6. Seminara SB, Achermann JC, Genel M, Jameson JL, Crowley WF, Jr. X-linked adrenal hypoplasia congenita: a mutation in DAX1 expands the phenotypic spectrum in males and females. J Clin Endocrinol Metab. 1999;84(12):4501-9.

7. Domenice S, Latronico AC, Brito VN, Arnhold IJ, Kok F, Mendonca BB. Adrenocorticotropin-dependent precocious puberty of testicular origin in a boy with X-linked adrenal hypoplasia congenita due to a novel mutation in the DAX1 gene. J Clin Endocrinol Metab. 2001;86(9):4068-71.

8. Yeste D, Gonzalez-Nino C, Perez de Nanclares G, Perez-Nanclares G, Audi L, Castano $\mathrm{L}$, et al. ACTH-dependent precocious pseudopuberty in an infant with DAX1 gene mutation. Eur J Pediatr. 2009;168(1):65-9.

9. Achermann JC, Ito M, Silverman BL, Habiby RL, Pang S, Rosler A, et al. Missense mutations cluster within the carboxyl-terminal region of DAX-1 and impair transcriptional repression. J Clin Endocrinol Metab. 2001;86(7):3171-5.

10. Lalli E, Bardoni B, Zazopoulos E, Wurtz JM, Strom TM, Moras D, et al. A transcriptional silencing domain in DAX-1 whose mutation causes adrenal hypoplasia congenita. Mol Endocrinol. 1997;11(13):1950-60.

11. Nakae J, Tajima T, Kusuda S, Kohda N, Okabe T, Shinohara N, et al. Truncation at the C-terminus of the DAX-1 protein impairs its biological actions in patients with Xlinked adrenal hypoplasia congenita. J Clin Endocrinol Metab. 1996;81(10):3680-5.

12. Peter M, Viemann M, Partsch CJ, Sippell WG. Congenital adrenal hypoplasia: clinical spectrum, experience with hormonal diagnosis, and report on new point mutations of the DAX-1 gene. J Clin Endocrinol Metab. 1998;83(8):2666-74. 


\section{Figure Legend}

\section{Fig. 1 Mutation analysis}

Electropherograms of directly sequenced PCR-amplified fragments from genomic DNA of the patients, his parents (mother and father) and a control showing a $C$ to $A$ exchange in

Exon 1 (position c.870), leading to the substitution of a cystein at position 290 with a premature stop codon (p.Cys290Ter). The mutation is hemizygous in the patient and heterozygous in the mother, as to be expected for a X-linked mode of inheritance.

\section{Fig. 2 Model of wild type and p.Cys290Stop DAX1 mutant proteins}

A. DAX1 consist of two domains, the $\mathrm{N}$-terminal DNA-binding domain and the C-terminal putative Ligand-binding Domain. Predicted wild-type DAX1 protein model.

B. The DAX1 p.Cys290 stop mutation leads to a loss of 180 amino acids, which in turn results in a predicted change in protein folding according to homology modelling. 
Figure 1

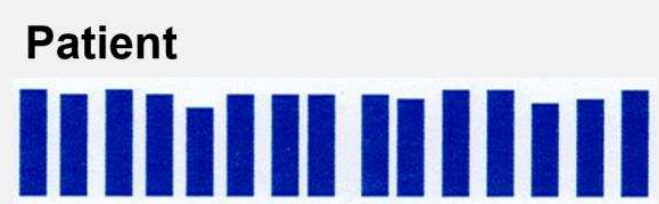

C G C A.A C T G A T G G G C G 190

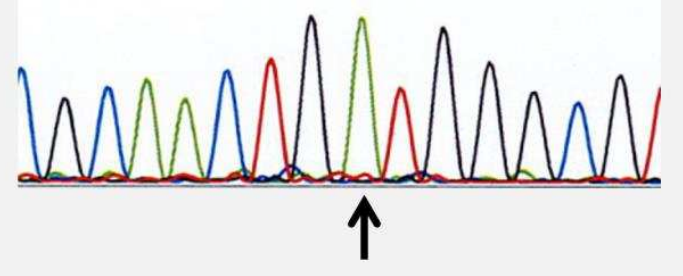

c. $870 \mathrm{C}>\mathrm{A}$

p.Cys290>Stop

Mother

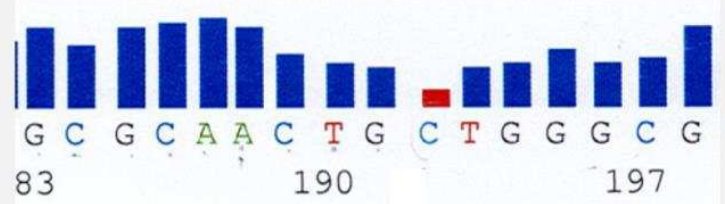

AnA c. 870 C>A / WT
Control

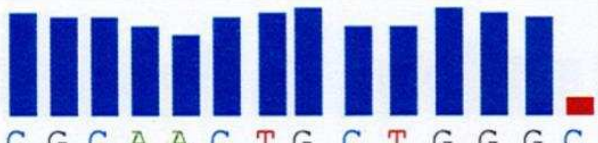

C G C A A C T G C T G G G C

190

197

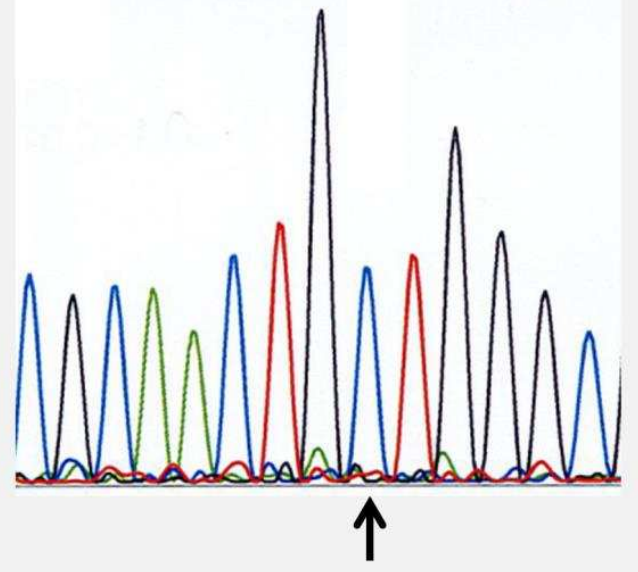

c. $870 \mathrm{C}$ (WT)

\section{Father}
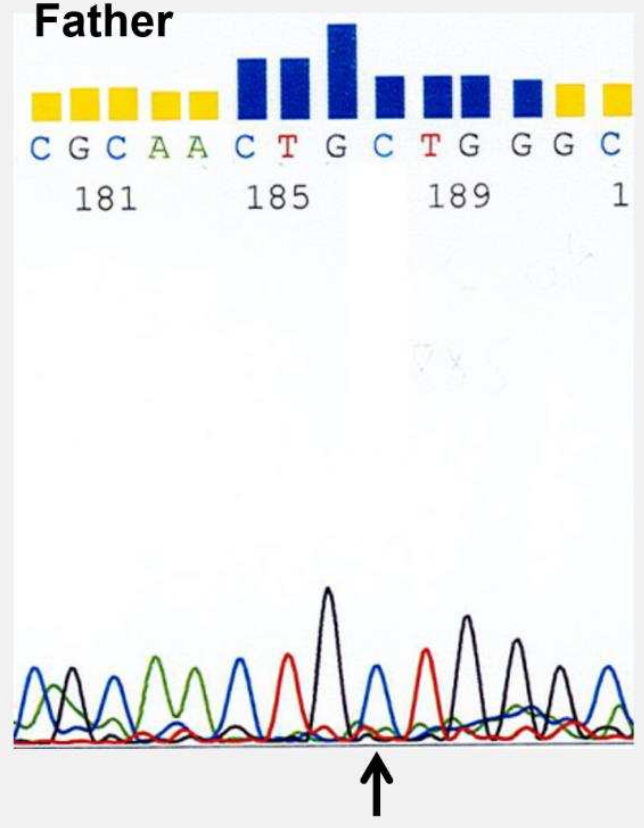

c. $870 \mathrm{C}$ (WT) 
Figure 2

A

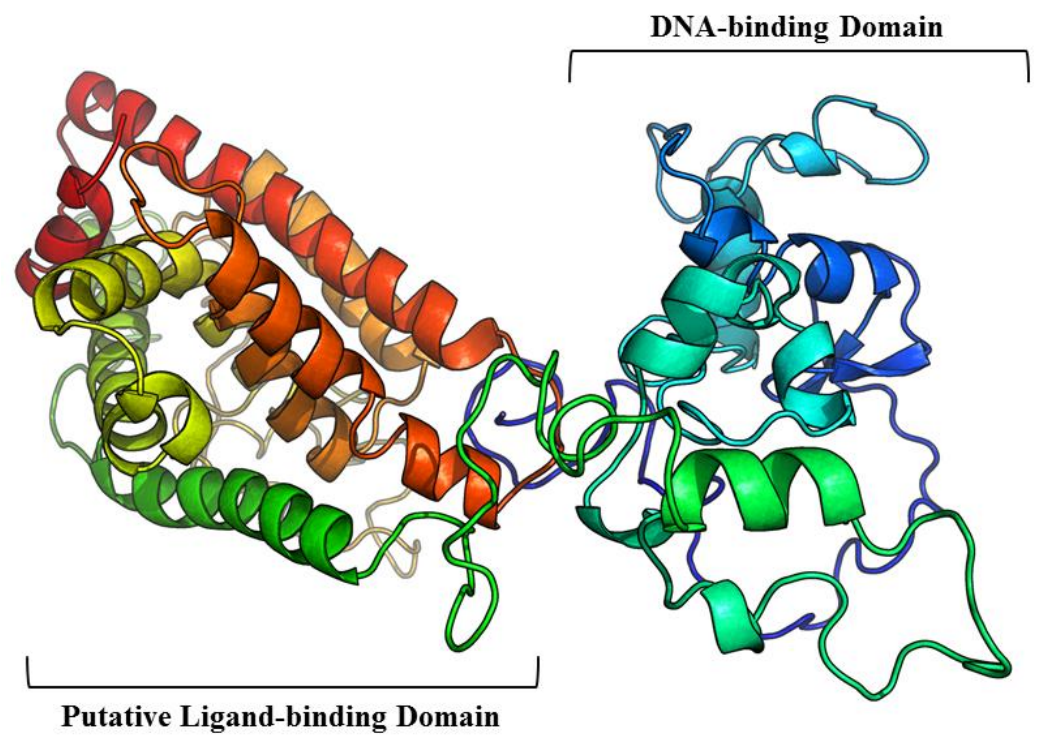

B

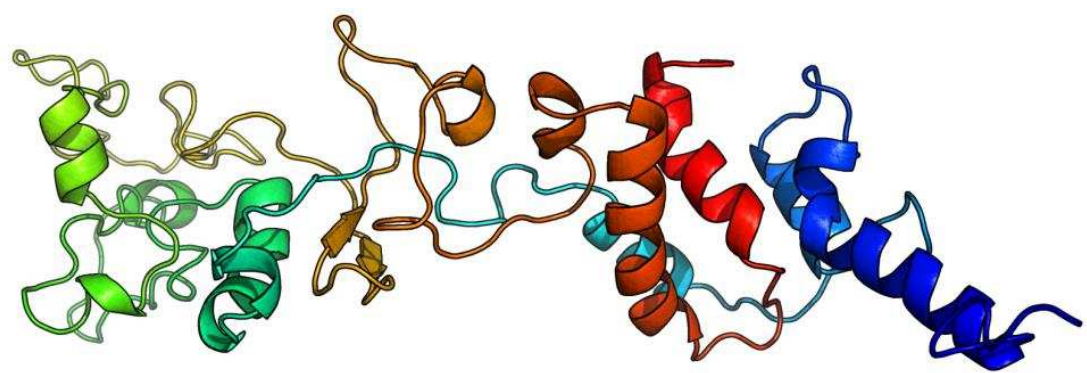

